\title{
Investigation of Cache Coherence Strategies in a Mobile Client/Server Environment
}

\author{
C.D.M. Berkenbrock and M.A.R. Dantas \\ Department of Informatics and Statistics (INE), \\ University of Santa Catarina (UFSC), \\ 88040-900 Florianopolis, Brazil \\ \{diacui, mario\}@inf.ufsc.br
}

\begin{abstract}
In this article, we present an investigation case study based on an implementation and performance analysis of three different cache coherence strategies over a real wireless environment. Our research work considers the broadcasting timestamp, the cache coherency schema with incremental update propagation and the amnesic terminals strategies. These strategies are based on periodic broadcast of invalidation reports. The performance of these strategies is analysed through an ordinary real environment. In this environment we compare the impact of invalidation report size, broadcast interval and cache size in mobile devices.
\end{abstract}

\section{Introduction}

Data replication in mobile devices can represent an interesting mechanism to reduce latency, data transfer from servers and collision avoidance in wireless environments. However, this approach can only be considered successful if users can find essential data in the local cache. As a result, mobile users can avoid the access to the wireless broadcast link. On the other hand, as disconnection is frequently observed in wireless configurations data stored verification in a mobile device is a complex task for several classes of applications.

In this article, we present an investigation case study based on an implementation and performance analysis research considering three different cache coherence strategies over a real wireless environment. The broadcasting timestamp (TS)[1], cache coherence schema with incremental update propagation (CCSIUP) [2] and amnesic terminals (AT) [1] strategies are based on a periodical broadcast of cache invalidation report (IR). Our experimental results reflect the impact of the size of IR and the cache size of mobile devices when utilizing a database application.

The paper is organized as follows. In section 2 we presented the experimental environment architecture. In section 3 we discuss the results obtained in a real wireless configuration. Finally, in section 4 we present conclusions of the research work. 


\section{Experimental Environment Architecture}

The client/server paradigm for distributed computing in wired networks is a well known architecture with many proposals for cache coherence [3]. This approach is also considered in the literature for wireless environments (e.g. $[4,5])$, however with special concern about disconnection and clients moving.

In this article, we consider the TS, CCS-IUP and AT techniques, because these strategies have invalidation report (IR) and stateless servers. IR is a lightweight mechanism for wireless networks that informs only the necessary items that were modified. The stateless servers are not obliged to keep information related to mobile devices in its area and it is not necessary to know about the cache of clients.

The environment architecture developed to execute our experiments is shown in figure 1. The architecture has three main components: a server, clients and a database system. In addition, we implemented the three protocols strategies, proposed in the literature.

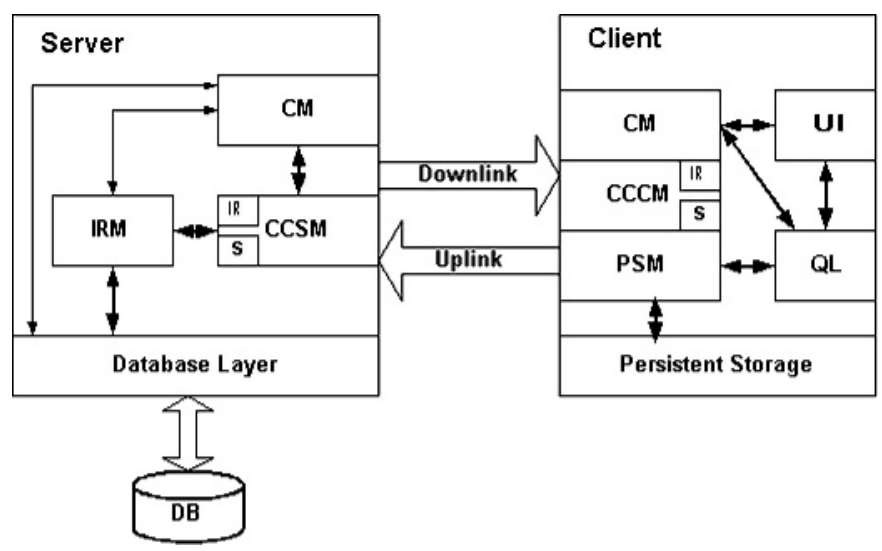

Fig. 1. The environment architecture

Inside the server the modules are communication manager $(\mathrm{CM})$, cache coherence server manager (CCSM) and invalidation report manager (IRM). CM is responsible for clients link management with the server side. The CCSM verifies which strategy is being used and adds the appropriated parameters inside the IR for this strategy. The IRM is responsible for the modification of the IR when the database is modified.

On the other hand, clients are characterized by the modules communication manager $(\mathrm{CM})$, cache coherence client manager $(\mathrm{CCCM})$, persistent storage manager (PSM), query list (QL) and user interface (UI). The CM client manages the connection with the server. The CCCM is responsible for receiving IRs from the server and applies the cache modification following a specific strategy (S). 
The PSM executes any modification necessary into the cache of the mobile device. The QL stores all the registers that are being modified and UI is a friendly interface to mobile users to access the environment.

The database system component consider for our experiments was the Mckoi SQL [6]. We employed this database, because it is open source JAVA SQL database system package and provides interesting functions to develop applications (e.g. trigger and referential integrity).

\section{$3 \quad$ Experimental Results}

In the server database we used 50 items, which were accessed by two mobile devices. The broadcast window used was 10 intervals, similar to others research works (e.g. [4, 5]), with 20 seconds between intervals. In addition, the experimental tests were executed using the TS, CCS-IUP and AT considering that mobile users have 5, 15 and 25 percent of item from the database locally cached.

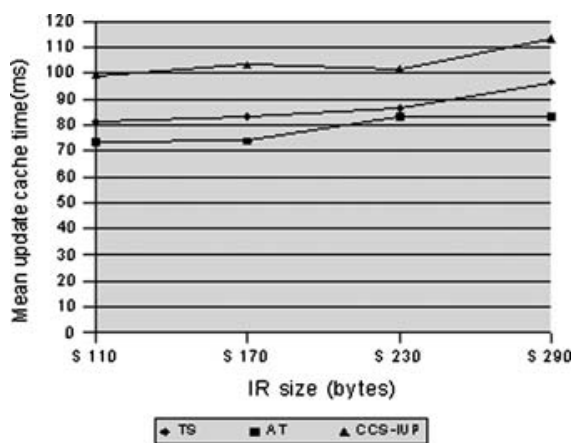

Fig. 2. Mean update cache time vs. IR size

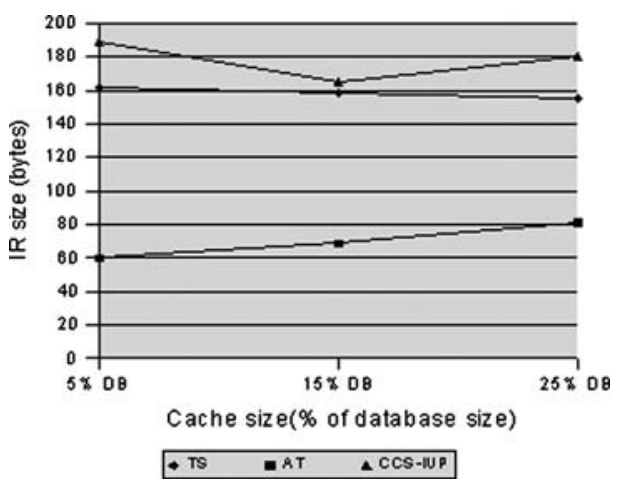

Fig. 4. IR size vs. Cache size

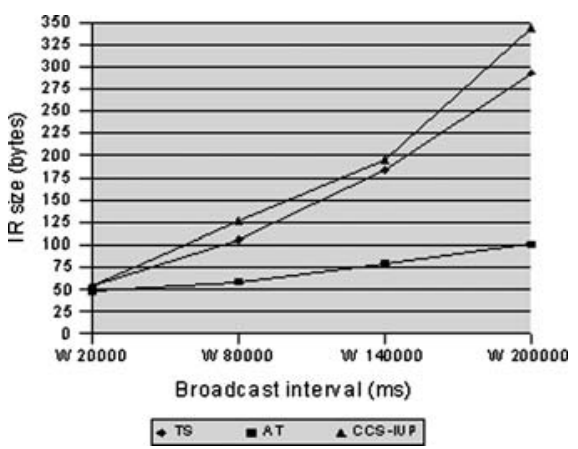

Fig. 3. IR size vs. Broadcast interval

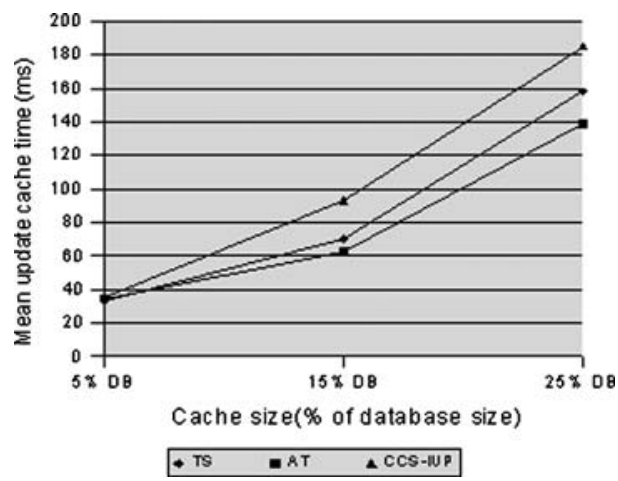

Fig. 5. Mean update cache time vs. Cache size 
In figure 2 our results indicates that the TS and CCS-IUP spend more time to update the cache than the AT approach. Figure 3 shows IR sizes related to broadcast intervals. The graphic shows that the TS and CCS-IUP have a tendency to increase IR sizes when broadcast intervals increase.

The relation of the notification size against the cache size is presented in figure 4. The increase of the cache size resulted in the increase of the IR size for AT. The TS registered a small decrement of the IR size and the CCS-IUP did not get a regular behavior. In figure 5, as we expected, it is possible to verified that the increase of the cache size directly implies in the time to update the data store in mobile clients.

\section{Conclusions}

In this paper we presented an investigation case study based on an implementation and performance analysis of three different strategies for cache coherence in wireless networks.

The experimental results demonstrated facilities and constrains of the strategies based on effects from the invalidation report (IR) size, broadcast interval and mobile clients cache size. The results demonstrated different performance for the parameters considered. The AT strategy had a interesting performance, but with less facilities compared to the other two approaches.

\section{Acknowledgements}

This research was partially supported by CNPQ (Brazilian National Research Council) under the grant 132145/2004-9.

\section{References}

1. Daniel Barbar; and Tomasz Imielnski. Sleepers and workaholics: caching strategies in mobile environments (extended version). The VLDB Journal, (1995) 4(4):567602.

2. Hyunsik Chung and Haengrae Cho. Data caching with incremental update propagation in mobile computing environments. In Australian Computer Journal (1998).

3. Magnus E. Bjornsson and Liuba Shrira. BuddyCache: high-performance object storage for collaborative strong-consistency applications in a WAN. Proceedings of the 17th ACM SIGPLAN conference on Object-oriented programming, systems, languages, and applications,(2002) 26-39.

4. Joanne Holliday and Divyakant Agrawal and Amr El Abbadi. Disconnection modes for mobile databases. Wirel. Netw, (2002) 391-402.

5. Jin Jing and Ahmed Elmagarmid and Abdelsalam Sumi Helal and Rafael Alonso. Bit-sequences: an adaptive cache invalidation method in mobile client/server environments. Mob. Netw. Appl, (1997) 115-127.

6. Mckoi SQL Database System. Available online: http://www.mckoi.com/database/. 